\title{
Role of Mitral Annular Systolic Velocity in The Evaluation of Left Atrial Appendage Function in Mitral Stenosis
}

\author{
AAS Majumder, MS Tanveer, AQM Reza, AW Chowdhury, M Shahabuddin \\ Department of Cardiology, National Institute of Cardiovascular Diseases, Dhaka
}

\begin{abstract}
Key words:

Mitral stenosis, Left atrial appendage function, Annular systolic velocity

Background : Mitral stenosis (MS) is the most common valvular heart disease. Thromboembolism is one of the most important complications of $M S$, especially when it is associated with atrial fibrillation (AF).Patients with sinus rhythm (SR) are also sussceptible to this complication when it is associated with left atrial appendage (LAA) dysfunction. LAA dysfunction is an independent predictor of thromboembolism in mitral stenosis.

Objectives: To investigate whether there is a relation between mitral annular velocities obtained by Doppler tissue imaging (DTI) and LAA function determined by transoesophageal echocardiography (TEE) and to determine if the annular velocities can predict the presence of the inactive LAA in MS.

Methods: Sixty MS patients were evaluated by transthoracic echocardiography and all patients underwent transesophageal echocardiography. The annular systolic (S-wave) and diastolic (Emand Am-waves) velocities were recorded. Inactive LAA was defined as LAA emptying velocity $<25$ $\mathrm{cm} / \mathrm{sec}$. Patients were divided into three groups; group $A I(n=18)$ : sinus rhythm $(S R)$ and $L A A$ emptying velocity e" $25 \mathrm{~cm} / \mathrm{sec}$, group AII $(n=22): S R$ and LAA emptying velocity $<25 \mathrm{~cm} / \mathrm{sec}$ and group $B(n=20)$ : atrial fibrillation.

Results: Thrombus was detected in 14 patients and spontaneous echo contrast (SEC) was detected in 48 patients. Both S-wave and peak LAA emptying velocities were decreasing, while SEC frequency and density were increasing from group $A$ to group $B$. There was a positive correlation between LAA emptying vs. S-wave and LAA emptying vs. Am velocities ( $p<0.001, r=0.708$ and $p<0.001, r=0.495)$. Multivariate regression analysis showed that only $S$-wave is the independent predictor of inactive LAA $(p=0.001$, odds ratio $=0.133,95 \% C I=0.032-0.556)$. In patients with $S R$, the cutoff value of $S$-wave was $14 \mathrm{~cm} / \mathrm{sec}$ for the prediction of the presence of inactive LAA (sensitivity: 92.3\%, specificity: 95.3\%).

Conclusions: $S$-wave is an independent predictor of inactive LAA and a useful parameter in estimating inactive LAA in MS with SR..
\end{abstract}

(CVJ 2008; 1(1) : 26-33)

\section{Introduction}

The left atrial appendage (LAA) is a long, tubular and trabecular structure and has a narrow junction with the left atrium (LA). The LAA has been the focus of clinicians' interests, because it is a potential site for the development of thrombus in several diseases. Normally, this highly dynamic structure prevents stasis, but when the LAA function is impaired, stasis will increase, which leads to formation of spontaneous echo contrast (SEC) and/ or thrombus. ${ }^{1-3}$ Furthermore, it has been shown that the presence of inactive LAA is an independent predictor of thromboembolic events. ${ }^{4-6}$ Hence, detection of the presence of inactive LAA can provide the opportunity for intervention such as anticoagulant therapy.
It is known that LAA velocities are reduced in mitral stenosis (MS). ${ }^{3-6}$ Recently, it has been reported that annular velocities obtained by tissue Doppler imaging (TDI) are also reduced in MS. ${ }^{7-9}$ The LAA has a close anatomic and functional relation with lateral annulus and thus annular velocities obtained by TDI may reflect the LAA functions. ${ }^{1}$ The aim of the study was to investigate whether there is a relation between mitral annular velocities obtained by TDI and LAA function and to determine if the annular velocities can predict the presence of the inactive LAA in patients with MS.

\section{Methods}

The study population consisted of 60 patients with isolated MS (41 females and 19 males; mean age: 30.5

Address Correspondence : Prof. A A S Majumder, Department of Cardiology, National Institute of Cardiovascular Diseases, aasm@dhaka.net 
\pm 8.2 years). Patients with any of the following were excluded from the study: hypertension, diabetes mellitus, evidence of coronary artery disease, aortic stenosis, moderate or severe mitral regurgitation, moderate or severe aortic regurgitation, history of mitral commissurotomy or valvuloplasty, congestive heart failure, and using antiplatelet or anticoagulant drug. Written informed consent was obtained from all the subjects.

\section{Transthoracic Echocardiography}

Transthoracic echocardiographic examinations were performed with Acuson (Siemens) with a $2-4$ $\mathrm{MHz}$ phased array transducer. The patients were studied in the left lateral position. Echocardiographic techniques and calculations of different cardiac dimensions were performed in accordance with the recommendations of The American Society of Echocardiography. ${ }^{10}$ Left ventricular ejection fraction (EF) was calculated by using modified Simpson's rule technique. Mitral valve area (MVA) was measured by planimetry in the parasternal short-axis view. The patients were graded according to MVA as mild $(1.50-2.50 \mathrm{~cm} 2)$, moderate (1.00-1.49 cm2), and severe $\mathrm{MS}(<1.0 \mathrm{~cm} 2)$. Transmitral mean gradient (MG) and peak gradient (PG) were measured by continuous wave Doppler.

\section{Doppler Tissue Imaging}

DTI was performed in the apical four chamber view and sample volume was placed in lateral wall. A Doppler velocity range of " 20 to $20 \mathrm{~cm} / \mathrm{sec}$ was selected. Three major velocities were recorded (Figure 1): one positive systolic velocity (S-wave) and two negative diastolic velocities (E- and A-waves). The peak systolic and diastolic velocities were measured at a sweep speed of $50 \mathrm{~mm} / \mathrm{sec}$.
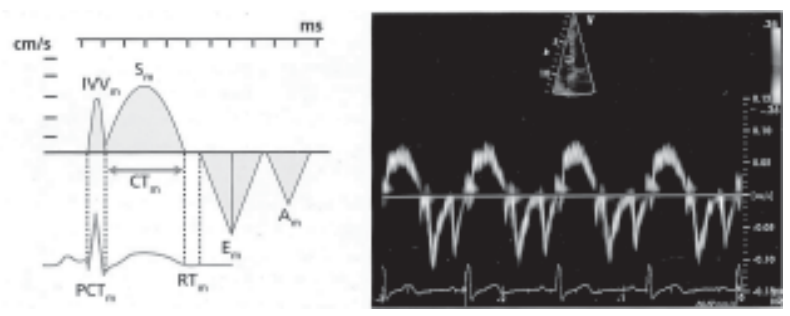

Fig.-1: Annular velocities obtained by DTI showing 3 main waves such as $S m=$ myocardial systolic velocity, $E m=$ Myocardial early diastolic velocity and Am= myocardial late diastolic velocity. Others parameters are: $I V V m=$ myocardial isovolumic velocity, $C T m=$ myocardial contraction time, $P C T m=$ myocardial precontraction time, $R T m=$ myocardial relaxation time (After Galderisi et al 2007).
Transesophageal Echocardiography

Multiplane transesophageal echocardiography (TEE) was performed in all patients. A one lead electrocardiogram was recorded continuously during the procedure. The LA and LAA imaging was begun in the horizontal plane, then the transducer was rotated progressively to $60 \%$ and $90 \%$; at the same degrees, images were also evaluated after slight and more pronounced counterclockwise rotation of the probe, rotation of the transducer to $110 æ \%$ and $130 æ \%$ was coupled with more pronounced counterclockwise rotation of the probe. Electronic rotation of the transducer to $180 æ \%$ was then performed. All images were recorded by zooming the LAA and optimizing the gain settings to minimize gray-noise artifacts.

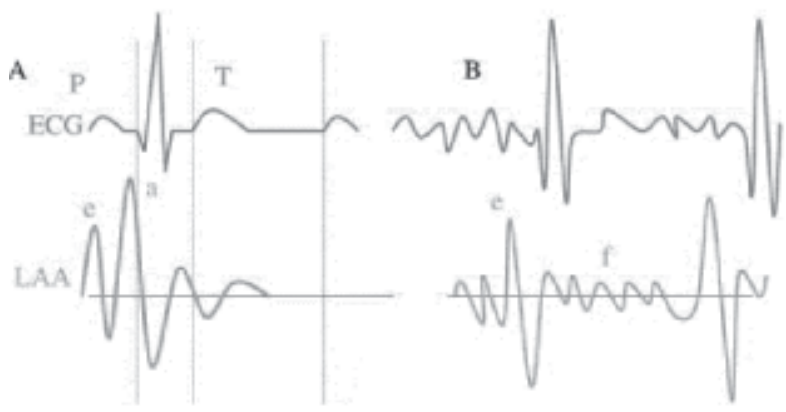

Fig.-2: Representation of LAA pulsed Doppler (ECGgated) recording in sinus rhythm (left, A) and atrial fibrillation (right, B). $e=$ passive LAA emptying flow, $a=$ active $L A A$ emptying flow and $f=$ passive $L A A$ emptying flow in AF (After Donal et al. 2005).

We examined the LAA in the short and long axis. LA thrombus was diagnosed by the presence of clearly defined echogenic intracavitary mass different from the underlying endocardium. SEC was diagnosed by the presence of dynamic smoke-like echoes in the LA cavity and LAA with a characteristic swirling motion. ${ }^{11}$ The severity of SEC was graded from 0 to $4+$ according to the following criteria: $0=$ none (absence of echogenicity); $1+=$ mild (minimal echogenicity located in the LAA or sparsely distributed in the main cavity of the LA, which may be detectable only transiently during the cardiac cycle and which is imperceptible at the operating gain settings for two-dimensional echocardiographic analysis); $2+=$ mild-to-moderate (more dense swirling pattern than $1+$ but with a similar distribution, detectable without increased gain settings); $3+=$ moderate (dense swirling pattern in 
the LAA, generally associated with somewhat lesser intensity in the main cavity; it may fluctuate in intensity but is constantly detectable throughout the cardiac cycle); $4+=$ severe (intense echo density and very slow swirling patterns in the LAA, usually with a similar density in the main cavity). ${ }^{12}$ The intra-observer and inter-observer variability were $5.2 \%$ and $8.1 \%$, respectively.

Artifact images were considered when a location and echogenicity suggestive of reverberations were obtained at different TEE rotational angles, in particular, artifacts were supposed to be found in the LAA twice as far from the transducer as an anatomical interface. The distances of each thrombus-like image from the transducer and from other anatomical interfaces were measured. The LAA flow profiles were obtained by placing the sample volume of the pulsed Doppler into the orifice of the appendage. Late peak emptying velocity was measured to represent LAA contractile function. Inactive LAA was defined as late peak LAA emptying velocity $<25 \mathrm{~cm} / \mathrm{sec}^{3,5,13}$ The patients were divided into three groups; group AI: sinus rhythm (SR) and late peak LAA emptying velocity e" $25 \mathrm{~cm} / \mathrm{sec}$, group AII: SR and late peak LAA emptying velocity $<25 \mathrm{~cm} / \mathrm{sec}$, and group $B$ : atrial fibrillation (AF).

\section{Statistical Analysis}

All calculations were performed with Statistical Package for the Social Sciences 10.0 (SPSS 10.0) program. Continuous variables between groups were compared with the one-way ANOVA test. Categorical variables were tested with the chi-square test. Multivariate, stepwise backward conditional logistic regression analysis was used to determine the relative importance of the independent predictors associated with inactive LAA in patients with SR. Age, LA dimension, MVA, MG, PG, and S-wave velocity were selected in the multivariate model. The Pearson correlation analysis was used to establish the association between S-wave and late peak LAA emptying velocities. A receiver operator characteristic (ROC) curve analysis was performed to identify the optimal cutoff point of S-wave velocity (at which sensitivity and specificity is maximal) to predict inactive LAA in patients with SR. The area under the curve (AUC) value was calculated as a measure of the accuracy of the test.

\section{Results}

Table-I

Baseline characteristic of the patients

\begin{tabular}{lccccc}
\hline & $\begin{array}{c}\text { Group A } \\
(\mathrm{n}=40)\end{array}$ & $\begin{array}{c}\text { Group B } \\
(\mathrm{n}=20)\end{array}$ & $p$-value \\
& Mean \pm SD & Mean & \pm SD & \\
\hline Age (years) & 28.8 & \pm 8.1 & 32.5 & \pm 8.3 & $0.100^{\mathrm{NS}}$ \\
Sex(M/F) & 13 & $/ 27$ & $6 /$ & 14 & $0.844^{\mathrm{NS}}$ \\
MVA (cm $\left.{ }^{2}\right)$ & 1.07 & \pm 0.21 & 0.77 & \pm 0.15 & $0.001^{\mathrm{S}}$ \\
LAD (mm) & 47.6 & \pm 10.0 & 54.2 & \pm 7.5 & $0.012^{\mathrm{S}}$ \\
EF $(\%)$ & 60.2 & \pm 5.9 & 58.1 & \pm 5.1 & $0.187^{\mathrm{NS}}$ \\
MG (mmHg) & 15.3 & \pm 5.9 & 11.1 & \pm 2.1 & $0.003^{\mathrm{S}}$ \\
PG (mmHg) & 22.9 & \pm 8.1 & 21.2 & \pm 3.9 & $0.364^{\mathrm{NS}}$ \\
S wave (cm/sec) & 17.5 & \pm 3.8 & 12.0 & \pm 1.1 & $0.001^{\mathrm{S}}$ \\
Em (cm/sec) & 14.4 & \pm 3.1 & 13.3 & \pm 3.1 & 0.194 \\
Am (cm/sec) & 14.5 & \pm 2.7 & - & & - \\
Laaev (cm/sec) & 21.6 & \pm 8.4 & 3.5 & \pm 5.8 & $0.001^{\mathrm{S}}$ \\
Mean SEC density & 2.1 & \pm 1.4 & 4.6 & \pm 0.9 & $0.001^{\mathrm{S}}$ \\
SEC (\%) & 23 & $(57.5)$ & 20 & $(100)$ & $0.001^{\mathrm{S}}$ \\
Thrombus (\%) & 2 & $(5.0)$ & 12 & $(60.0)$ & $0.001^{\mathrm{S}}$ \\
\hline
\end{tabular}

MVA = mitral valve area $\mathrm{LAD}=$ left atrial dimension; $\mathrm{EF}=$ Ejection fraction; $\mathrm{MG}=$ mean gradient across mitral orifice; $\mathrm{PG}=$ peak gradient across mitral orifice S-wave $=$ peak annular systolic velocity; $\mathrm{Em}=$ peak annular early diastolic velocity; $\mathrm{Am}=$ peak annular late diastolic velocity; Laaev = left atrial appendage emptying velocity; $\mathrm{SEC}=$ spontaneous echo-contrast; Group A: Sinus Rhythm $(n=40)$; Group B: Atrial fibrillation $(n=20) ; p<0.05$ is significant; $S=$ significant; $\mathrm{NS}=$ not significant.

\section{Baseline characteristics of the Patients :}

Most of the patients had severe MS . Mean MVA was $0.92 \pm 0.21 \mathrm{~cm}^{2}$. AF was present in 20 patients (33.33\%). As expected, the patients had larger LA dimension. Systolic and diastolic annular velocities were significantly reduced in patients compared to the controls. However, age, gender, and EF were similar in patients (Table I).

Demographic and Transthoracic Echocardiographic Characteristics of Groups:

The mean age and LA dimension were progressively increasing from group AI to group B. Both group AII and group B had narrower MVA than group AI. In group AII patients, $M G$ and $P G$ were significantly higher than group-AI and group B. Gender and EF were similar among the groups (Table-II). 
Table-II

Demographic and Transthoracic Echocardiographic Characteristics of Patient Groups

\begin{tabular}{|c|c|c|c|c|c|c|c|c|c|}
\hline & \multicolumn{2}{|c|}{$\begin{array}{l}\text { Group A I } \\
\quad(n=18)\end{array}$} & \multicolumn{2}{|c|}{$\begin{array}{l}\text { Group AII } \\
\quad(\mathrm{n}=22)\end{array}$} & \multicolumn{2}{|c|}{$\begin{array}{c}\text { Group B } \\
(\mathrm{n}=20)\end{array}$} & \multirow[t]{2}{*}{$p 1$} & \multirow[t]{2}{*}{$p 2$} & \multirow[t]{2}{*}{ p3 } \\
\hline & Mean & $\pm \mathrm{SD}$ & Mean & $\pm \mathrm{SD}$ & Mean & $\pm \mathrm{SD}$ & & & \\
\hline$\overline{\text { Age (years) }}$ & 30.6 & \pm 8.0 & 27.3 & \pm 8.1 & 32.5 & \pm 8.3 & $0.203^{\mathrm{NS}}$ & $0.473^{\mathrm{NS}}$ & $0.05^{\mathrm{NS}}$ \\
\hline $\operatorname{Sex}(M / F)$ & $5 /$ & 13 & $8 /$ & 14 & $6 /$ & 14 & $0.564^{\mathrm{NS}}$ & $0.880^{\mathrm{NS}}$ & $0.662^{\mathrm{NS}}$ \\
\hline $\operatorname{MVA}(\mathrm{cm} 2)$ & 1.26 & \pm 0.15 & 0.91 & \pm 0.07 & 0.77 & \pm 0.15 & $0.001^{\mathrm{S}}$ & $0.001^{\mathrm{S}}$ & $0.001^{\mathrm{S}}$ \\
\hline $\mathrm{LAD}(\mathrm{mm})$ & 40.4 & \pm 3.5 & 53.4 & \pm 9.8 & 54.2 & \pm 7.5 & $0.001^{\mathrm{S}}$ & $0.001^{\mathrm{S}}$ & $0.787^{\mathrm{NS}}$ \\
\hline $\mathrm{EF}(\%)$ & 60.5 & \pm 5.5 & 59.8 & \pm 6.3 & 58.1 & \pm 5.1 & $0.775^{\mathrm{NS}}$ & $0.180^{\mathrm{NS}}$ & $0.312^{\mathrm{NS}}$ \\
\hline $\mathrm{MG}(\mathrm{mmHg})$ & 9.8 & \pm 3.4 & 19.9 & \pm 2.7 & 11.1 & \pm 2.1 & $0.001^{\mathrm{S}}$ & $0.172^{\mathrm{NS}}$ & $0.001^{\mathrm{S}}$ \\
\hline $\mathrm{PG}(\mathrm{mmHg})$ & 15.4 & \pm 3.9 & 29.9 & \pm 4.6 & 21.2 & \pm 3.9 & $0.001^{\mathrm{S}}$ & $0.001^{\mathrm{S}}$ & $0.001^{\mathrm{S}}$ \\
\hline
\end{tabular}

Group A I (n=18): LAA EV e" $25 \mathrm{~cm} / \mathrm{sec}$; Group A II (n=22): LAA EV < $25 \mathrm{~cm} / \mathrm{sec}$. Group B (n=20): AF.

p1= Group A I versus Group A II; p2= Group A I versus Group B; p3= Group A II versus Group B; p < 0.05 is significant; S $=$ significant; $\mathrm{NS}=$ not significant.

Table-III

DTI and Transesophageal Echocardiographic Variables in Groups $(n=60)$ :

\begin{tabular}{|c|c|c|c|c|c|c|c|c|c|}
\hline & \multicolumn{2}{|c|}{$\begin{array}{c}\text { Group A I } \\
(\mathrm{n}=18)\end{array}$} & \multicolumn{2}{|c|}{$\begin{array}{c}\text { Group A II } \\
(\mathrm{n}=22)\end{array}$} & \multicolumn{2}{|c|}{$\begin{array}{c}\text { Group B } \\
(\mathrm{n}=20)\end{array}$} & \multirow[t]{2}{*}{$p 1$} & \multirow[t]{2}{*}{$p 2$} & \multirow[t]{2}{*}{ p3 } \\
\hline & Mean & $\pm \mathrm{SD}$ & Mean & $\pm \mathrm{SD}$ & Mean & $\pm \mathrm{SD}$ & & & \\
\hline S wave $(\mathrm{cm} / \mathrm{sec})$ & 19.6 & \pm 2.7 & 15.8 & \pm 3.8 & 12.0 & \pm 1.1 & $0.001^{\mathrm{S}}$ & $0.001^{\mathrm{S}}$ & $0.000^{\mathrm{S}}$ \\
\hline $\operatorname{Em}(\mathrm{cm} / \mathrm{sec})$ & 14.3 & \pm 2.7 & 14.5 & \pm 3.3 & 13.3 & \pm 3.1 & $0.807 \mathrm{NS}$ & $0.317^{\mathrm{NS}}$ & $0.234^{\mathrm{NS}}$ \\
\hline $\operatorname{Am}(\mathrm{cm} / \mathrm{sec})$ & 15.8 & \pm 1.2 & 13.4 & \pm 3.2 & - & & $0.006^{\mathrm{S}}$ & - & - \\
\hline Laaev (cm/sec) & 29.5 & \pm 5.8 & 15.1 & \pm 2.7 & - & & $0.001^{\mathrm{S}}$ & - & - \\
\hline Mean SEC density & y 1.5 & \pm 1.1 & 2.6 & \pm 1.5 & 4.6 & \pm 0.9 & $0.009^{\mathrm{S}}$ & $0.001^{\mathrm{S}}$ & $0.001^{\mathrm{S}}$ \\
\hline SEC (\%) & 3 & $(16.7)$ & 20 & $(90.9)$ & 20 & $(100)$ & $0.001^{\mathrm{S}}$ & $0.001^{\mathrm{S}}$ & $0.268^{\mathrm{NS}}$ \\
\hline Thrombus & - & & 2 & $(9.1)$ & 12 & $(60.0)$ & $0.296^{\mathrm{NS}}$ & $0.003^{\mathrm{S}}$ & $0.022^{\mathrm{S}}$ \\
\hline
\end{tabular}

Group A I (n=18): LAA EV e" 25 cm/sec; Group A II (n=22): LAA EV < 25 cm/sec; Group B (n=20): AF

p1= Group A I versus Group A II; p2= Group A I versus Group B; p3= Group A II versus Group B; p < 0.05 is significant; S= significant; $\mathrm{NS}=$ not significant.

DTI and Transesophageal Echocardiographic Variables of Groups

The patients with SR had biphasic LAA flow pattern and those with AF had no definite wave pattern. Inactive LAA was observed in 22 patients with SR. Both S-wave Am and peak LAA emptying velocities were decreasing, while SEC frequency and density were increasing from group AI to group B. Annular E-wave was similar in all groups. Thrombi were found in 14 of whom 2 in group AII and 12 in group $B$ patients; 10 of group B were within the LAA and the remaining was within both the LA main cavity and the LAA. However, there was no thrombus in group AI. SEC was detected in 48 patients (80\%) and the intensity of SEC was as follows: 0 (17 patients), $1+$ (1 patient), $2+$ (8 patients), $3+(7$ patients), and $4+$ (11 patients) (Table III).

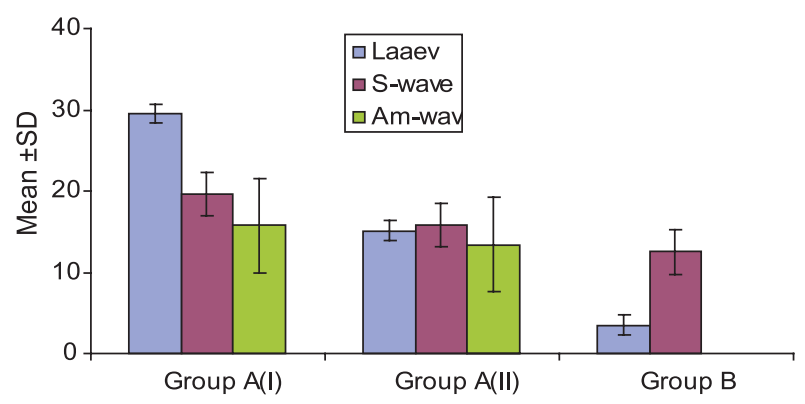

Fig.-3: Correlation between $L A A E V$, S-wave and Am $(\mathrm{cm} / \mathrm{sec})$. 
We found a significant positive correlation between LAA emptying vs. S-wave velocities and LAA emptying vs. Am velocities by using the Pearson correlation analysis $(p<0.001, r=0.708$ and $p<0.001, r=495$ (Fig. 4 and 5). But there were no correlation between LAA emptying velocity and Em-wave. Age, LA dimension, MVA, $\mathrm{MG}, \mathrm{PG}$, and S-wave velocity were evaluated by multivariate regression analysis with backward elimination.

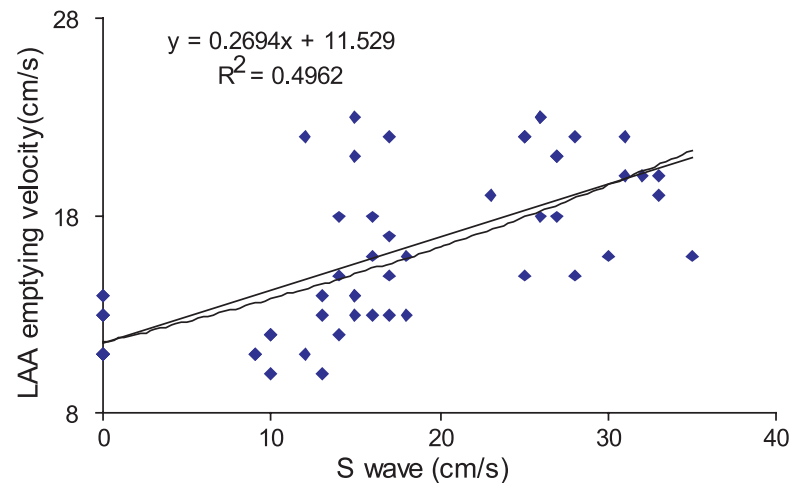

Fig.-4: Significant correlationship $(p<0.001$; $r=0.708)$ between $S$-wave $(\mathrm{cm} / \mathrm{s})$ and LAA emptying velocity $(\mathrm{cm} / \mathrm{s})$.

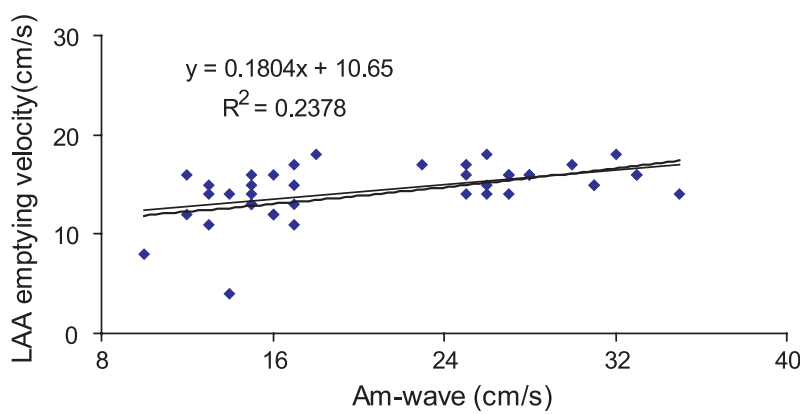

Fig.-5: Significant correlationship $(p<0.001$; $r=0.495)$ between Am-wave $(\mathrm{cm} / \mathrm{s})$ and $L A A$ emptying velocity.

Only S-wave velocity was found to be an independent predictor for the presence of inactive LAA $(p=0.001$, odds ratio $=0.133,95 \% \mathrm{CI}=0.032-0.556)$. In patients with SR, the cutoff value of S-wave velocity was $14 \mathrm{~cm} / \mathrm{sec}$, obtained by the ROC curve analysis, for prediction of the presence of inactive LAA with a sensitivity of $92.3 \%$ and a specificity of $95.3 \%$. The AUC was calculated to be 0.905 ( $p=0.001,95 \%$ CI 0.825-0.985). Figure 6 shows the ROC curve of Swave velocity.

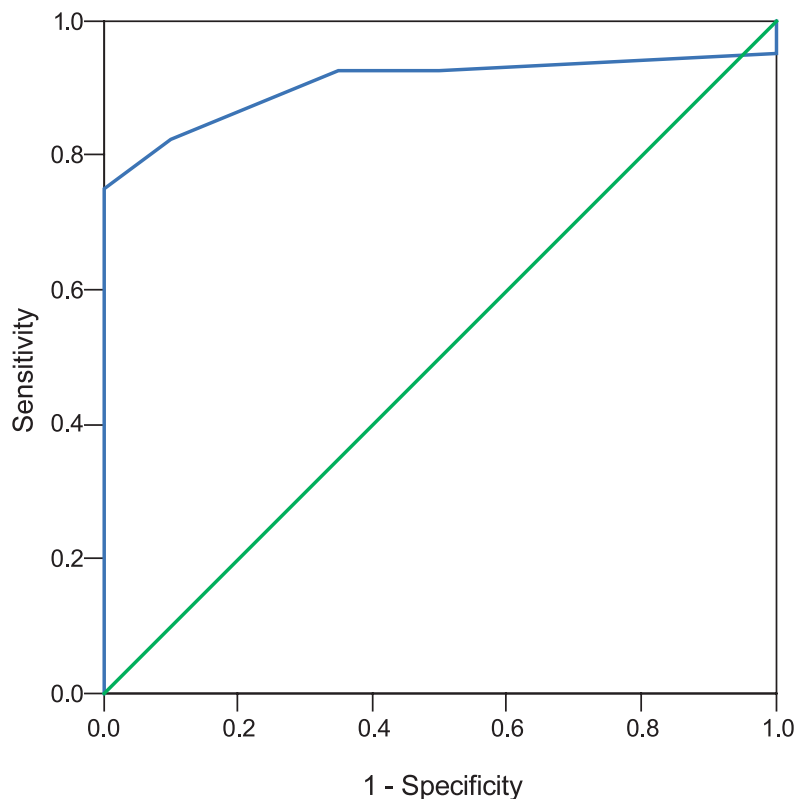

Fig.-6: The ROC curve of $S$-wave for predicting inactive left atrial appendage in mitral stenosis patients with sinus rhythm. The magnitude of the area under the curve indicates the accuracy of the test. $R O C=$ receiver operator characteristic.

\section{Discussion}

Our results clearly showed that there is a close relationship between LAA emptying and annular systolic and late diastolic velocities.

Sinus Rhythm

The mitral annulus has an important role for LA and left ventricular (LV) functions by moving throughout LV long axis. ${ }^{14-16}$ The LV long-axis function which is a useful echocardiographic index of LV systolic function can be evaluated by measuring excursion of the mitral annulus obtained by M-mode echocardiography or by measuring annular systolic velocity obtained by TDI. ${ }^{7,9}$ The excursion of the mitral annulus accounted for approximately $20 \%$ of the total LV filling and emptying in healthy subjects. ${ }^{14}$ Furthermore, mitral annular movement contributes to LA filling by creating a suction effect during systole and it contributes to LA emptying by decreasing LA volume during diastole. ${ }^{14-16}$

In MS patients, LV, LA, and LAA functions are impaired and the excursion of mitral annulus is 
reduced due to scarring and inflammatory processes. $^{3-8}$ It has been recently reported that annular velocities obtained by DTI are reduced in patients with MS. ${ }^{7-9}$ We also showed that annular velocities are reduced in MS.

In our study, there was a positive correlation between annular systolic and late diastolic velocities with LAA emptying velocity and reduction in annular velocities were corresponded closely with reduction in LAA emptying velocity. Furthermore, a reduction in annular velocities corresponded with an increase in SEC frequency and density. Thus, the annular systolic and late diastolic velocity alterations reflected the changes in LAA function. Normally, quadriphasic (especially at slow heart rates) or biphasic pattern of blood flow within the LAA is obtained. The initial LAA emptying and filling flows occur passively. After these waves, more important late diastolic LAA emptying and filling waves, resulting from active contraction and relaxation of the LAA, are seen. ${ }^{1,3,5,17,18}$

The LAA flow pattern and velocities are influenced by changes in LV and LA function. ${ }^{1,17-22}$ It was reported that marked elevation of LA pressures may reduce LAA emptying velocity and lead to thrombus formation in LAA. ${ }^{19}$ Hoit et al. ${ }^{20}$ showed the effects of altered loading conditions on the LAA flow velocities. They found a significant reduction in the early emptying velocity and a nonsignificant reduction in the late emptying velocity when LA pressure was increased by phenylephrine infusion. They also clearly showed that LV systolic function is a major determinant of the late LAA emptying velocity. Bilge et al. ${ }^{21}$ evaluated the untreated hypertensive patients with SR to clarify the effect of elevated afterload on LAA functions and they suggest that marked elevation of afterload imposed on the LA and LAA might play an important role in decreased LAA emptying velocity and formation of LA SEC and thrombus. But they did not research the effect of annular velocities on LAA function.

In this study, group AII patients have narrower MVA, higher MG and PG, and lower annular systolic and late diastolic velocities than group AI. However, multivariate regression analysis showed that only annular systolic velocity that reflects $\mathrm{LV}$ long-axis function is an independent predictor of the inactive LAA. So this result showed that impaired LV long-axis function may be the most important factor that contributes to the LAA dysfunction in MS patients with SR.

TEE is a gold standard method to assess the LAA function. ${ }^{1,23,24}$ However, this technique is semiinvasive and unsuitable for repeated examinations. Firstly in our study, it has been researched whether the annular systolic velocity can predict the presence of the inactive LAA in these patient groups. The cutoff value of annular S-wave velocity obtained by ROC curve analysis was $14 \mathrm{~cm} / \mathrm{sec}$ for the prediction of the presence of inactive LAA with a sensitivity of $92.3 \%$ and a specificity of $95.3 \%$. Both sensitivity and specificity were very high. Therefore, the annular systolic velocity might be used as a good variable in the estimation of LAA function in MS patients. The previous studies reported that $0-13 \%$ of MS patients with SR have thrombi. ${ }^{4,25,26}$ In our study, there were only 22 patients in group AII and we demonstrated only 2 thrombi in this group. This may be largely related to our smaller number of cases.

\section{Atrial Fibrillation}

Garcia-Fernandez et al. ${ }^{27}$ described two different types of LAA flow patterns in AF patients: active sawtooth pattern and no identifiable wave. They found that second pattern has a higher incidence of LAA SEC and thrombus than sawtooth pattern and SR. We observed no identifiable wave pattern in all patients with AF possibly due to severe MS. Group $B$ patients were older and have narrower MVA and larger LA. Furthermore, group B patients had lower LAA emptying and annular systolic velocities than group AI and group AII. Both lower LAA emptying and annular systolic and late diastolic velocities are associated with significant stasis, which led to higher density of SEC and higher prevalence of SEC and thrombus in LAA in group B patients. For this reason, MS patients with $\mathrm{AF}$ have higher thromboembolic risk. Similarly, it was found that patients with MS and AF have a 17 -fold increased risk of stroke than age-matched controls in the Framingham Heart Study. ${ }^{28}$

\section{Clinical Implications}

There is a consensus to anticoagulant therapy, with a target international normalized ratio between 2.5 and 3.5 in MS patients with AF. ${ }^{29,30}$ But there is no such consensus to consider anticoagulation in MS patients with SR. European Society of 
Cardiology recommended that decision of anticoagulant therapy in MS patients with SR should be made on the risk benefit ratio of long term. ${ }^{30}$ We detected the inactive LAA in $92.3 \%$ of patients with SR and low annular systolic velocity $(<14 \mathrm{~cm} / \mathrm{sec})$. It is known that the presence of inactive LAA is an independent predictor of the development of SEC, thrombus, and finally thromboembolic events. ${ }^{4-6}$ So, we suggest that anticoagulant therapy should be started in all MS patients who have SR and low annular systolic velocity $(<14 \mathrm{~cm} / \mathrm{sec})$.

\section{Study Limitations}

Various parameters related to LA pressure, atrial stiffness, and body function could also be selected in the multivariate model. Other populations of MS may have different cutoff values, such as patients with hypertension or coronary artery disease may have higher cutoff level and those with mitral regurgitation may have lower cutoff level. Study subjects might be compared with healthy control. Sample size contains smaller number of cases. These data must be checked with further studies.

\section{Conclusions}

The systolic and diastolic annular velocities obtained by TDI are reduced in patients with MS. There is a positive correlation between annular systolic and LAA emptying velocities in MS. The annular systolic velocity is an independent predictor for the presence of inactive LAA in MS patients with SR. Finally, lower annular systolic velocity could be considered as an indication for anticoagulation in MS patients with SR.

\section{References:}

1. Al-Saady NM, Obel OA, Camm AJ: Left atrial appendage: Structure, function and role in thromboembolism. Heart 1999; 82: 547-555.

2. Gurlertop Y, Yilmaz M, Acikel M, et al: Tissue Doppler properties of the left atrial appendage in patients with mitral valve disease. Echocardiography 2004; 21(4): 319-324.

3. Daimee MA, Salama AL, Cherian G, et al: Left atrial appendage function in mitral stenosis: Is a group in sinus rhythm at risk of thromboembolism? Int J Cardiol 1998; 66(1): 45-54.

4. Li YH, Hwang JJ, Ko YL, et al: Left atrial spontaneous echo contrast in patients with rheumatic mitral valve disease in sinus rhythm. Implication of an altered left atrial appendage function in its formation. Chest 1995; 108(1): 99-103.
5. Eryol NK, Topsakal R, Kiranatli B, et al: Color Doppler tissue imaging to evaluate left atrial appendage function in mitral stenosis. Echocardiography 2003; 20(1): 2935 .

6. Mahilmaran A, Nayar PG, Sudarsana G, et al: Relationship of left atrial appendage function to left ventricular function. Indian Heart J 2004; 56(4): 293298.

7. O” zer N, Can I, Atalar E, et al: Left ventricular long axis function is reduced in patients with rheumatic mitral stenosis. Echocardiography 2004; 21: 107-112.

8. O” zdemir K, Altunkeser BB, Go"k H, et al: Does the myocardial performance index affect pulmonary artery pressure in patients with mitral stenosis? A tissue Doppler imaging study. Echocardiography 2003; 20: 249-256.

9. Sengupta PP, Mohan JC, Mehta V, et al: Effects of percutaneous mitral commissurotomy on longitudinal left ventricular dynamics in mitral stenosis: Quantitative assessment by tissue velocity imaging. $\mathrm{J} \mathrm{Am} \mathrm{Soc}$ Echocardiogr 2004; 17(8): 824-828.

10. Schiller NB, Shah PM, Crawford M, et al: Recommendations for quantitation of the left ventricle by two dimensional echocardiography. American Society of Echocardiography Committee on Standards, Subcommittee on Quantitation of Two-Dimensional Echocardiograms. J Am Soc Echocardiogr 1989; 2(5): 358-367.

11. Goswami KC, Yadav R, Rao MB, et al: Clinical and echocardiographic predictors of left atrial clot and spontaneous echo contrast in patient with severe rheumatic mitral stenosis: A prospective study in 200 patients by transesophageal echocardiography. Int $J$ Cardiol 2000; 73: 273-279.

12. Fatkin D, Kelly RP, Feneley MP: Relations between left atrial appendage blood flow velocity, spontaneous echocardiographic contrast and thromboembolic risk in vivo. J Am Coll Cardiol 1994; 23(4): 961-969.

13. Bollmann A, Binias K, Grothues F, et al: Left atrial appendage flow in nonrheumatic atrial fibrillation: Relationship with pulmonary venous flow and ECG Fibrillatory wave amplitude. Chest 2001; 119(2): 485492.

14. Emilsson K: Right ventricular long-axis function in relation to left ventricular systolic function. Clin Physiol Funct Imaging 2004; 24(4): 179-248.

15. Yip GW, Zhang Y, Tan PY, et al: Left ventricular longaxis changes in early diastole and systole: Impact of systolic function on diastole. Clin Sci (Lond) 2002; 102: $515-522$.

16. Henein MY, Gibson DG: Normal long axis function. Heart 1999; 81: 111-113.

17. Stollberger C, Schneider B, Finsterer J: Elimination of the left atrial appendage to prevent stroke or embolism? 
Anatomic, physiologic, and Pathophysiologic considerations. Chest 2003; 124(6): 2356-2362.

18. Agmon Y, Khandheria BK, Meissner I, et al: Are left atrial appendage flow velocities adequate surrogates of global left atrial function? A population-based transthoracic and transesophageal echocardiographic study. J Am Soc Echocardiogr 2002; 15(5): 433-440.

19. Tabata T, Oki T, Fukuda N, et al: Influence of left atrial pressure on left atrial appendage flow velocity patterns in patients in sinus rhythm. J Am Soc Echocardiogr 1996; 9: 857-864.

20. Hoit BD, Shao Y, Gabel M: Influence of acutely altered loading conditions on left atrial appendage flow velocity. J Am Coll Cardiol 1994; 24: 1117-1123.

21. Bilge M, Eryonucu B, Guler N, et al: Transesophageal echocardiographic assessment of left atrial appendage function in untreated systemic hypertensive patients with sinus rhythm. J Am Soc Echocardiogr 2000; 13: 271-276.

22. G* urlertop Y, Yilmaz M, Ac,ikel M, et al: The use of anatomic M-Mode echocardiography to determine the left atrial appendage functions in patients with sinus rhythm. Echocardiography 2005; 22(2): 99-103.

23. Maltagliati A, Pepi M, Tamborini G, et al: Usefulness of multiplane transesophageal echocardiography in the recognition of artifacts and normal anatomical variants that may mimic left atrial thrombi in patients with atrial fibrillation. Ital Heart J 2003; 4(11): 797-802.

24. Agmon Y, Khandheria BK, Gentile F, et al: Echocardiographic assessment of the left atrial appendage. J Am Coll Cardiol 1999; 34(7): 1867-1877.

25. Saidi SJ, Motamedi MH: Incidence and factors influencing left atrial clot in patients with mitral stenosis and normal sinus rhythm. Heart 2004; 90: 1342-1343.

26. Golbasi Z, Cicek D, Canbay A, et al: Left atrial appendage function in patients with mitral stenosis in sinus rhythm. Eur J Echocardiogr 2002; 3: 39-43.

27. Garcia-Fernandez MA, Torrecilla EG, San Roman D, et al: Left atrial appendage Doppler flow patterns. Implication on thrombus formation. Am Heart J 1992; 124: 955-961.

28. Wolf PA, Dawber TR, Thomas HE, et al: Epidemiologic assessment of chronic atrial fibrillation and risk of stroke: The Framingham study. Neurology 1978; 28(10): 973-977.

29. Bonow RO, Carabello B, DeLeon AC, et al: ACC/AHA Task Force Report. ACC/AHA guidelines for the management of patients with valvular heart disease. $J$ Am Coll Cardiol 1998; 32: 1486-1588.

30. Lung B, Gohlke-Barwolf C, Tornos P, et al: Working Group on Valvular Heart Disease. Recommendations on the management of the asymptomatic patient with valvular heart disease. Eur Heart J 2002; 23(16): 1252-1266. 